\title{
Excited State Intramolecular Proton Transfer in 2-(2'-Arylsulfon- amidophenyl)benzimidazole Derivatives: Insights into the Origin of Donor Substituent-Induced Emission Energy Shifts
}

\author{
Yonggang Wu, PaDreyia V. Lawson, Maged M. Henary, Karin Schmidt, \\ Jean-Luc Brédas, and Christoph J. Fahrni* \\ School of Chemistry and Biochemistry and Petit Institute for Bioengineering \\ and Bioscience, Georgia Institute of Technology, 901 Atlantic Drive, \\ Atlanta, Georgia 30332-0400
}

\section{List of Contents}

Table S1: $\quad$ Selected solvent parameters utilized in the solvatochromic shift analyses

\section{Summary of Spectroscopic Data (Tables S2-S6)}

Table S2: $\quad$ Peak absorption and emission energies of compound 1a

Table S3: $\quad$ Peak absorption and emission energies of compound 2a

Table S4: Peak absorption and emission energies of compound 3a

Table S5: $\quad$ Peak absorption and emission energies of compound 4a

Table S6: $\quad$ Peak absorption and emission energies of compound 5a

\section{Summary of Optimized Geometries (Table S7-S18)}

Table S7: Cartesian atomic coordinates for TPBI ( $\mathrm{S}_{0}$, DFT-RB3LYP/6-31G(d))

Table S8: Cartesian atomic coordinates for TPBI $\left(S_{0}, R H F / 3-21+G(d, p)\right)$

Table S9: Cartesian atomic coordinates for TPBI $\left(\mathrm{S}_{0}, \mathrm{RHF} / 6-31+\mathrm{G}(\mathrm{d}, \mathrm{p})\right)$

Table S10: Cartesian atomic coordinates for compound 1c $\left(S_{0}, R H F / 3-21+G(d, p)\right)$

Table S11: Cartesian atomic coordinates for compound 2c $\left(S_{0}, R H F / 3-21+G(d, p)\right)$

Table S12: Cartesian atomic coordinates for compound 3c $\left(\mathrm{S}_{0}, \mathrm{RHF} / 3-21+\mathrm{G}(\mathrm{d}, \mathrm{p})\right)$

Table S13: Cartesian atomic coordinates for the phototautomer of compound 1c $\left(\mathrm{S}_{0}, \mathrm{RHF} / 3-21+\mathrm{G}(\mathrm{d}, \mathrm{p})\right)$

Table S14: Cartesian atomic coordinates for the phototautomer of compound 2c $\left(\mathrm{S}_{0}, \mathrm{RHF} / 3-21+\mathrm{G}(\mathrm{d}, \mathrm{p})\right)$ 
Table S15: Cartesian atomic coordinates for the phototautomer of compound 3c $\left(\mathrm{S}_{0}, \mathrm{RHF} / 3-21+\mathrm{G}(\mathrm{d}, \mathrm{p})\right)$

Table S16: Cartesian atomic coordinates for the phototautomer of compound 1c $\left(\mathrm{S}_{1}, \mathrm{RHF}-\mathrm{CIS} / 3-21+\mathrm{G}(\mathrm{d}, \mathrm{p})\right)$

Table S17: Cartesian atomic coordinates for the phototautomer of compound $\mathbf{2 c}$ $\left(\mathrm{S}_{1}, \mathrm{RHF}-\mathrm{CIS} / 3-21+\mathrm{G}(\mathrm{d}, \mathrm{p})\right)$

Table S18: Cartesian atomic coordinates for the phototautomer of compound $\mathbf{3 c}$ $\left(\mathrm{S}_{1}, \mathrm{RHF}-\mathrm{CIS} / 3-21+\mathrm{G}(\mathrm{d}, \mathrm{p})\right)$

\section{Summary of Structural Parameters for the Optimized Geometries (Table S19-S21)}

Table S19: Selected interatomic distances $(\AA)$ for geometry-optimized structures.

Table S20: Selected bond angles (deg) for geometry-optimized structures.

Table S21: Selected dihedral angles (deg) for geometry-optimized structures.

\section{Summary of Computed Photophysical Properties (Tables S22-S30)}

Table S22: Computed vertical excitation energies (eV) and photophysical properties for 1c-3c (INDO-SCI//DFT-RB3LYP/6-31G(d)).

Table S23: Computed vertical excitation energies $(\mathrm{eV})$ and photophysical properties for $\mathbf{1 c}-\mathbf{3 c}$ (INDO-SCI//RHF/3-21+G(d,p)).

Table S24: Computed vertical excitation energies $(\mathrm{eV})$ and photophysical properties for $\mathbf{1 c}-\mathbf{3 c}$ (INDO-SCI//RHF/3-21+G(d,p)).

Table S25: Computed vertical excitation energies $(\mathrm{eV})$ and photophysical properties for $\mathbf{1 c}-\mathbf{3 c}$ (INDO-SCI(SCRF $\mathrm{H}_{2} \mathrm{O}$ )//RHF/3-21+G(d,p)).

Table S26: Computed vertical excitation energies (eV) and photophysical properties for 1c-3c (INDO-SCI(SCRF MeOH)//RHF/3-21+G(d,p)).

Table S27: Computed vertical emission energies (eV) and photophysical properties for $\mathbf{1 c}-\mathbf{3 c}$ (INDO-SCI/RHF-CIS/3-21+G(d,p)).

Table S28: Computed vertical emission energies $(\mathrm{eV})$ and photophysical properties for $\mathbf{1 c}-\mathbf{3 c}$ (INDO-SCI//RHF-CIS/3-21+G(d,p)).

Table S29: Computed vertical emission energies $(\mathrm{eV})$ and photophysical properties for $\mathbf{1 c}-\mathbf{3 c}$ (INDO-SCI(SCRF $\mathrm{H}_{2} \mathrm{O}$ )//RHF-CIS/3-21+G(d,p)).

Table S30: Computed vertical emission energies (eV) and photophysical properties for $\mathbf{1 c}-\mathbf{3 c}$ (INDO-SCI(SCRF MeOH)//RHF-CIS/3-21+G(d,p)).

\section{Summary of Plotted Densities and Isosurfaces}

Table S31: Selected molecular orbital isosurfaces and electron attachment/detachment densities for 1c-3c (RHF-CIS/3-21+G(d,p) optimized geometries). 
Table S1: Selected solvent parameters utilized in the solvatochromic shift analyses

\begin{tabular}{llllllll}
\hline No. & Solvent & $\varepsilon_{r}$ & $n$ & $E_{\mathrm{T}} \mathrm{N}$ & $\pi^{*}$ & $\alpha$ & $\beta$ \\
\hline 1 & 1,4-dioxane & 2.27 & 1.422 & 0.164 & 0.490 & 0.000 & 0.370 \\
2 & di-n-butylether & 3.18 & 1.398 & 0.071 & 0.180 & 0.000 & 0.460 \\
3 & diethyl ether & 4.42 & 1.352 & 0.117 & 0.240 & 0.000 & 0.470 \\
4 & tetrahdyrofuran & 7.47 & 1.406 & 0.207 & 0.550 & 0.000 & 0.550 \\
5 & $n$-butylacetate & 5.01 & 1.394 & 0.241 & 0.529343 & 0.000 & 0.450 \\
6 & ethylacetate & 6.053 & 1.372 & 0.228 & 0.450 & 0.000 & 0.450 \\
7 & chloroform & 4.89 & 1.446 & 0.259 & 0.690 & 0.200 & 0.100 \\
8 & 1,2-dichloroethane & 10.74 & 1.445 & 0.327 & 0.730 & 0.000 & 0.100 \\
9 & dichloromethane & 24.56 & 1.424 & 0.309 & 0.730 & 0.130 & 0.100 \\
10 & $n$-butanenitrile & 24.56 & 1.383 & 0.364 & 0.630 & 0.000 & 0.400 \\
11 & acetonitrile & 35.94 & 1.344 & 0.460 & 0.660 & 0.190 & 0.400 \\
12 & $n$-butanol & 17.51 & 1.3993 & 0.586 & 0.36173 & 0.840 & 0.840 \\
13 & ethanol & 24.55 & 1.36143 & 0.654 & 0.340535 & 0.860 & 0.750 \\
14 & methanol & 32.04 & 1.3284 & 0.762 & 0.345582 & 0.980 & 0.660 \\
15 & water & 78 & 1.33 & 1.000 & 1.09 & 1.100 & 0.180 \\
\hline
\end{tabular}

\section{Summary of Spectroscopic Data (Tables S2-S6)}

The reported absorption energies refer to the peak of the lowest energy band. Fluorescence peak emission energies are corrected as described in the experimental section. All spectra were acquired at $298 \mathrm{~K}$.

Table S2: Peak absorption and emission energies of compound 1a

\begin{tabular}{llll}
\hline No. & Solvent & $v_{\mathrm{A}}\left[\mathrm{cm}^{-1}\right]$ & $v_{\mathrm{F}}\left[\mathrm{cm}^{-1}\right]$ \\
\hline 1 & 1,4-dioxane & 31443 & 20408 \\
2 & di-n-butylether & 31443 & 20408 \\
3 & diethyl ether & 31552 & 20534 \\
4 & tetrahdyrofuran & 31443 & 20408 \\
5 & $n$-butylacetate & 31443 & 20492 \\
6 & ethylacetate & 31552 & 20492 \\
7 & chloroform & 33327 & 20619 \\
8 & 1,2-dichloroethane & 33449 & 20450 \\
9 & dichloromethane & 33327 & 20408 \\
10 & $n$-butanenitrile & 31552 & 20576 \\
11 & acetonitrile & 31739 & 20833 \\
12 & $n$-butanol & 31645 & 21322 \\
13 & ethanol & 31739 & 21322 \\
14 & methanol & 31850 & 21322 \\
\hline
\end{tabular}


Table S3: Peak absorption and emission energies of compound 2a

\begin{tabular}{llll}
\hline No. & Solvent & $v_{\mathrm{A}}\left[\mathrm{cm}^{-1}\right]$ & $v_{\mathrm{F}}\left[\mathrm{cm}^{-1}\right]$ \\
\hline 1 & 1,4-dioxane & 33650 & 18490 \\
2 & di-n-butylether & 33270 & 18580 \\
3 & diethyl ether & 33400 & 18540 \\
4 & tetrahdyrofuran & 33120 & 18570 \\
5 & $n$-butylacetate & 33340 & 18600 \\
6 & ethylacetate & 33400 & 18650 \\
7 & chloroform & 33610 & 18760 \\
8 & 1,2-dichloroethane & 33530 & 18570 \\
9 & dichloromethane & 33380 & 18620 \\
10 & $n$-butanenitrile & 33170 & 18890 \\
11 & acetonitrile & 33350 & 19080 \\
12 & $n$-butanol & 33630 & 19590 \\
13 & ethanol & 33660 & 19550 \\
14 & methanol & 33670 & 19540 \\
\hline
\end{tabular}

Table S4: Peak absorption and emission energies of compound 3a

\begin{tabular}{llll}
\hline No. & Solvent & $v_{\mathrm{A}}\left[\mathrm{cm}^{-1}\right]$ & $\nu_{\mathrm{F}}\left[\mathrm{cm}^{-1}\right]$ \\
\hline 1 & 1,4-dioxane & 31800 & 21430 \\
2 & di-n-butylether & 31360 & 21540 \\
3 & diethyl ether & 31530 & 21460 \\
4 & tetrahdyrofuran & 31630 & 21470 \\
5 & $n$-butylacetate & 31480 & 21500 \\
6 & ethylacetate & 31570 & 21530 \\
7 & chloroform & 31890 & 21700 \\
8 & 1,2-dichloroethane & 31850 & 21520 \\
9 & dichloromethane & 31310 & 21530 \\
10 & $n$-butanenitrile & 31490 & 21680 \\
11 & acetonitrile & 31670 & 21910 \\
12 & $n$-butanol & 31840 & 22540 \\
13 & ethanol & 31995 & 22460 \\
14 & methanol & 32060 & 22460 \\
\hline
\end{tabular}


Table S5: Peak absorption and emission energies of compound 4a

\begin{tabular}{llll}
\hline No. & Solvent & $v_{\mathrm{A}}\left[\mathrm{cm}^{-1}\right]$ & $v_{\mathrm{F}}\left[\mathrm{cm}^{-1}\right]$ \\
\hline 1 & 1,4-dioxane & & 19240 \\
2 & di-n-butylether & 33340 & 19270 \\
3 & diethyl ether & 33450 & 19230 \\
4 & tetrahdyrofuran & & 19260 \\
5 & $n$-butylacetate & 33410 & 19260 \\
6 & ethylacetate & 33520 & 19330 \\
7 & chloroform & & 19490 \\
8 & 1,2-dichloroethane & & 19310 \\
9 & dichloromethane & 33340 & 19300 \\
10 & $n$-butanenitrile & 33280 & 19440 \\
11 & acetonitrile & 33400 & 19650 \\
12 & $n$-butanol & & 20140 \\
13 & ethanol & & 20020 \\
14 & methanol & & 20110 \\
\hline
\end{tabular}

Table S6: Peak absorption and emission energies of compound 5a

\begin{tabular}{llll}
\hline No. & Solvent & $v_{\mathrm{A}}\left[\mathrm{cm}^{-1}\right]$ & $v_{\mathrm{F}}\left[\mathrm{cm}^{-1}\right]$ \\
\hline 1 & 1,4-dioxane & 30400 & 20940 \\
2 & di-n-butylether & 30165 & 21040 \\
3 & diethyl ether & 30310 & 20990 \\
4 & tetrahdyrofuran & 30580 & 20980 \\
5 & $n$-butylacetate & 30260 & 20990 \\
6 & ethylacetate & 30320 & 21060 \\
7 & chloroform & 31270 & 21150 \\
8 & 1,2-dichloroethane & 30790 & 21070 \\
9 & dichloromethane & 30490 & 21060 \\
10 & $n$-butanenitrile & 30270 & 21220 \\
11 & acetonitrile & 30500 & 21400 \\
12 & $n$-butanol & 30670 & 21980 \\
13 & ethanol & 30590 & 21930 \\
14 & methanol & 30830 & 21910 \\
\hline
\end{tabular}




\section{Summary of Optimized Geometries (Table S7-S18)}

To exclude artifacts related to optimizing a local rather than global energy minimum structure, calculations were initiated from several starting geometries. Structures for which the optimization algorithm converged were checked for imaginary frequencies. Geometry optimized Cartesian atomic coordinates and distances are reported in Angstroms $(\AA)$. All energies are reported in atomic units (a.u.) unless otherwise noted. The corresponding level of theory is indicated. " $P T$ " is used to indicate the imino phototautomer form of the compound. "Para" indicates substitution at the 5- position and "meta" indicates substitution at the 4 - position (numbering scheme in Chart 1 ). " $S_{0}$ " corresponds to a singlet ground state calculation and " $S_{1}$ " corresponds to the first excited singlet state. 
Table S7: Cartesian atomic coordinates for TPBI ( $\mathrm{S}_{0}$, DFT-RB3LYP/6-31G(d), $\mathrm{E}=-1485.2238937845$ a.u.)

\begin{tabular}{|c|c|c|c|}
\hline Atom & $X(\AA)$ & $\mathrm{Y}(\AA)$ & $\mathrm{Z}(\AA)$ \\
\hline C & 3.044714 & 0.246025 & -0.65646 \\
\hline $\mathrm{C}$ & 3.722276 & 1.09556 & -1.53983 \\
\hline $\mathrm{C}$ & 4.902606 & 1.683755 & -1.09857 \\
\hline $\mathrm{C}$ & 5.407945 & 1.437216 & 0.19414 \\
\hline $\mathrm{C}$ & 4.751182 & 0.591542 & 1.085088 \\
\hline $\mathrm{C}$ & 3.567904 & 0.004014 & 0.635452 \\
\hline $\mathrm{N}$ & 2.66688 & -0.86742 & 1.221377 \\
\hline $\mathrm{N}$ & 1.86425 & -0.45477 & -0.82381 \\
\hline $\mathrm{C}$ & 1.656655 & -1.10814 & 0.308242 \\
\hline C & 0.514395 & -1.97901 & 0.596989 \\
\hline C & 0.554433 & -2.84214 & 1.70773 \\
\hline $\mathrm{C}$ & -0.52304 & -3.64822 & 2.048785 \\
\hline C & -1.6725 & -3.6208 & 1.254723 \\
\hline $\mathrm{C}$ & -1.73033 & -2.81107 & 0.126264 \\
\hline $\mathrm{C}$ & -0.65296 & -1.97809 & -0.21812 \\
\hline $\mathrm{N}$ & -0.70143 & -1.18914 & -1.38338 \\
\hline $\mathrm{S}$ & -2.01627 & -0.22102 & -1.83795 \\
\hline $\mathrm{O}$ & -3.22382 & -1.05014 & -1.84059 \\
\hline $\mathrm{O}$ & -1.543 & 0.463021 & -3.0407 \\
\hline $\mathrm{C}$ & -2.20909 & 1.001615 & -0.53806 \\
\hline $\mathrm{C}$ & -1.46456 & 2.182592 & -0.59415 \\
\hline $\mathrm{C}$ & -1.58962 & 3.113328 & 0.433528 \\
\hline $\mathrm{C}$ & -2.44831 & 2.886263 & 1.519803 \\
\hline $\mathrm{C}$ & -3.18575 & 1.695462 & 1.54798 \\
\hline C & -3.07641 & 0.752582 & 0.526957 \\
\hline $\mathrm{H}$ & 3.326514 & 1.280543 & -2.53356 \\
\hline $\mathrm{H}$ & 5.44988 & 2.347795 & -1.76159 \\
\hline $\mathrm{H}$ & 6.332635 & 1.916311 & 0.502998 \\
\hline $\mathrm{H}$ & 5.14734 & 0.401676 & 2.078644 \\
\hline $\mathrm{H}$ & 2.685232 & -1.18405 & 2.178252 \\
\hline $\mathrm{H}$ & 1.460036 & -2.90049 & 2.305756 \\
\hline $\mathrm{H}$ & -0.46142 & -4.30242 & 2.913168 \\
\hline $\mathrm{H}$ & -2.51962 & -4.25582 & 1.498608 \\
\hline $\mathrm{H}$ & -2.60265 & -2.81509 & -0.5152 \\
\hline $\mathrm{H}$ & 0.191021 & -0.72236 & -1.60224 \\
\hline $\mathrm{H}$ & -0.80963 & 2.371381 & -1.43835 \\
\hline $\mathrm{H}$ & -1.01392 & 4.034841 & 0.389455 \\
\hline $\mathrm{H}$ & -3.8616 & 1.503573 & 2.377699 \\
\hline $\mathrm{H}$ & -3.66371 & -0.15881 & 0.545333 \\
\hline $\mathrm{C}$ & -2.59581 & 3.91689 & 2.613772 \\
\hline $\mathrm{H}$ & -3.32801 & 4.685743 & 2.333915 \\
\hline $\mathrm{H}$ & -2.93949 & 3.464012 & 3.549215 \\
\hline $\mathrm{H}$ & -1.64812 & 4.429385 & 2.810405 \\
\hline
\end{tabular}


Table S8: Cartesian atomic coordinates for TPBI $\left(\mathrm{S}_{0}, \mathrm{RHF} / 3-21+\mathrm{G}(\mathrm{d}, \mathrm{p})\right.$, $E=-1470.1328205321$ a.u.)

\begin{tabular}{|c|c|c|c|}
\hline Atom & $X(\AA)$ & $Y(\AA)$ & $\mathrm{Z}(\AA)$ \\
\hline C & -2.098599 & 1.340056 & -1.020949 \\
\hline C & -3.068039 & 1.225576 & -2.006128 \\
\hline $\mathrm{C}$ & -4.378575 & 1.061403 & -1.601954 \\
\hline C & -4.718894 & 1.017956 & -0.241025 \\
\hline $\mathrm{C}$ & -3.760296 & 1.140498 & 0.747536 \\
\hline $\mathrm{C}$ & -2.447274 & 1.294572 & 0.329464 \\
\hline $\mathrm{N}$ & -1.251149 & 1.446994 & 1.022187 \\
\hline $\mathrm{N}$ & -0.709227 & 1.508035 & -1.131434 \\
\hline C & -0.246114 & 1.567275 & 0.085651 \\
\hline C & 1.161287 & 1.72675 & 0.465737 \\
\hline C & 1.497551 & 2.452059 & 1.604436 \\
\hline $\mathrm{C}$ & 2.820475 & 2.594621 & 1.98321 \\
\hline C & 3.822402 & 2.007658 & 1.225605 \\
\hline C & 3.501277 & 1.290989 & 0.085036 \\
\hline C & 2.178114 & 1.1505 & -0.298396 \\
\hline $\mathrm{N}$ & 1.856547 & 0.364005 & -1.452482 \\
\hline S & 1.943466 & -1.264963 & -1.424766 \\
\hline $\mathrm{O}$ & 3.24492 & -1.631443 & -0.913762 \\
\hline $\mathrm{O}$ & 1.509962 & -1.702799 & -2.73181 \\
\hline $\mathrm{C}$ & 0.74747 & -1.762355 & -0.240466 \\
\hline C & -0.551923 & -2.028678 & -0.650309 \\
\hline $\mathrm{C}$ & -1.505552 & -2.370331 & 0.289147 \\
\hline $\mathrm{C}$ & -1.177686 & -2.456202 & 1.64018 \\
\hline C & 0.130899 & -2.198069 & 2.031026 \\
\hline C & 1.096907 & -1.856177 & 1.096889 \\
\hline $\mathrm{H}$ & -2.798218 & 1.268883 & -3.04293 \\
\hline $\mathrm{H}$ & -5.154621 & 0.971523 & -2.337116 \\
\hline $\mathrm{H}$ & -5.748533 & 0.897792 & 0.036541 \\
\hline $\mathrm{H}$ & -4.025704 & 1.117968 & 1.787082 \\
\hline $\mathrm{H}$ & -1.120962 & 1.352251 & 2.004955 \\
\hline $\mathrm{H}$ & 0.726662 & 2.931774 & 2.176818 \\
\hline $\mathrm{H}$ & 3.067505 & 3.164147 & 2.857923 \\
\hline $\mathrm{H}$ & 4.850012 & 2.114767 & 1.51385 \\
\hline $\mathrm{H}$ & 4.262195 & 0.828049 & -0.508371 \\
\hline $\mathrm{H}$ & 1.13829 & 0.737293 & -2.055119 \\
\hline $\mathrm{H}$ & -0.806085 & -1.975891 & -1.691091 \\
\hline $\mathrm{H}$ & -2.509667 & -2.581378 & -0.029428 \\
\hline $\mathrm{H}$ & 0.406275 & -2.282529 & 3.065812 \\
\hline $\mathrm{H}$ & 2.111475 & -1.687286 & 1.401978 \\
\hline C & -2.224484 & -2.890441 & 2.645481 \\
\hline $\mathrm{H}$ & -2.462109 & -3.941353 & 2.510111 \\
\hline $\mathrm{H}$ & -1.875747 & -2.755804 & 3.66221 \\
\hline $\mathrm{H}$ & -3.142155 & -2.326058 & 2.518689 \\
\hline
\end{tabular}


Table S9: Cartesian atomic coordinates for TPBI $\left(\mathrm{S}_{0}, \mathrm{RHF} / 6-31+\mathrm{G}(\mathrm{d}, \mathrm{p})\right.$, $\mathrm{E}=-1477.8700109981$ a.u.)

\begin{tabular}{|c|c|c|c|}
\hline Atom & $X(\AA)$ & $Y(\AA)$ & $Z(\AA)$ \\
\hline $\mathrm{C}$ & -2.75234 & 0.728285 & -0.79202 \\
\hline $\mathrm{C}$ & -3.65925 & 0.319134 & -1.76674 \\
\hline $\mathrm{C}$ & -4.88408 & -0.15847 & -1.34644 \\
\hline $\mathrm{C}$ & -5.2101 & -0.22839 & 0.016877 \\
\hline $\mathrm{C}$ & -4.32164 & 0.180094 & 0.992347 \\
\hline $\mathrm{C}$ & -3.09201 & 0.658602 & 0.556425 \\
\hline $\mathrm{N}$ & -1.98794 & 1.146449 & 1.222767 \\
\hline $\mathrm{N}$ & -1.47279 & 1.233795 & -0.92354 \\
\hline $\mathrm{C}$ & -1.05531 & 1.459787 & 0.27697 \\
\hline $\mathrm{C}$ & 0.264872 & 2.013986 & 0.642782 \\
\hline $\mathrm{C}$ & 0.380339 & 2.780392 & 1.803111 \\
\hline $\mathrm{C}$ & 1.594223 & 3.305378 & 2.201061 \\
\hline C & 2.718849 & 3.080723 & 1.421014 \\
\hline $\mathrm{C}$ & 2.62196 & 2.351385 & 0.250298 \\
\hline $\mathrm{C}$ & 1.404709 & 1.807308 & -0.14702 \\
\hline $\mathrm{N}$ & 1.330799 & 1.092738 & -1.3656 \\
\hline $\mathrm{S}$ & 2.169218 & -0.29474 & -1.65007 \\
\hline $\mathrm{O}$ & 3.54708 & -0.0447 & -1.36079 \\
\hline $\mathrm{O}$ & 1.746648 & -0.70469 & -2.9519 \\
\hline $\mathrm{C}$ & 1.597157 & -1.49609 & -0.48235 \\
\hline $\mathrm{C}$ & 0.425368 & -2.19757 & -0.7423 \\
\hline $\mathrm{C}$ & -0.02958 & -3.12268 & 0.176807 \\
\hline $\mathrm{C}$ & 0.671901 & -3.36744 & 1.358445 \\
\hline $\mathrm{C}$ & 1.842058 & -2.65748 & 1.596117 \\
\hline C & 2.310381 & -1.72386 & 0.681606 \\
\hline $\mathrm{H}$ & -3.40545 & 0.377567 & -2.80946 \\
\hline $\mathrm{H}$ & -5.6065 & -0.48252 & -2.07384 \\
\hline $\mathrm{H}$ & -6.17449 & -0.60525 & 0.307128 \\
\hline $\mathrm{H}$ & -4.57642 & 0.130142 & 2.035968 \\
\hline $\mathrm{H}$ & -1.83066 & 1.11971 & 2.202031 \\
\hline $\mathrm{H}$ & -0.49671 & 2.989419 & 2.388989 \\
\hline $\mathrm{H}$ & 1.658389 & 3.895754 & 3.096936 \\
\hline $\mathrm{H}$ & 3.669091 & 3.492917 & 1.709436 \\
\hline $\mathrm{H}$ & 3.482678 & 2.19461 & -0.36881 \\
\hline $\mathrm{H}$ & 0.411171 & 1.047096 & -1.76403 \\
\hline $\mathrm{H}$ & -0.11227 & -2.02834 & -1.65647 \\
\hline $\mathrm{H}$ & -0.93533 & -3.66639 & -0.02839 \\
\hline $\mathrm{H}$ & 2.402696 & -2.83587 & 2.496741 \\
\hline $\mathrm{H}$ & 3.224936 & -1.19207 & 0.863068 \\
\hline $\mathrm{C}$ & 0.173708 & -4.40283 & 2.338145 \\
\hline $\mathrm{H}$ & 0.321355 & -5.40399 & 1.943931 \\
\hline $\mathrm{H}$ & 0.699299 & -4.33583 & 3.28323 \\
\hline $\mathrm{H}$ & -0.88693 & -4.2786 & 2.529949 \\
\hline
\end{tabular}


Table S10: Cartesian atomic coordinates for compound 1c ( $\mathrm{S}_{0}, \mathrm{RHF} / 3-21+\mathrm{G}(\mathrm{d}, \mathrm{p})$, $\mathrm{E}=-1544.5817551329$ a.u.)

\begin{tabular}{|c|c|c|c|}
\hline Atom & $X(\AA)$ & $Y(\AA)$ & $\mathrm{Z}(\AA)$ \\
\hline $\mathrm{C}$ & 1.803277 & 2.068823 & 0.429773 \\
\hline C & 2.682417 & 2.551143 & 1.388218 \\
\hline $\mathrm{C}$ & 4.033156 & 2.359386 & 1.174312 \\
\hline C & 4.500869 & 1.703952 & 0.024439 \\
\hline $\mathrm{C}$ & 3.632556 & 1.229237 & -0.940238 \\
\hline C & 2.278618 & 1.421581 & -0.711161 \\
\hline $\mathrm{N}$ & 1.143391 & 1.088087 & -1.443121 \\
\hline $\mathrm{N}$ & 0.40061 & 2.110658 & 0.384075 \\
\hline $\mathrm{C}$ & 0.049681 & 1.52793 & -0.727813 \\
\hline $\mathrm{C}$ & -1.325533 & 1.32804 & -1.196273 \\
\hline $\mathrm{C}$ & -1.613482 & 1.380382 & -2.556362 \\
\hline $\mathrm{C}$ & -2.904347 & 1.177352 & -3.011032 \\
\hline $\mathrm{C}$ & -3.921917 & 0.916591 & -2.106129 \\
\hline $\mathrm{C}$ & -3.649835 & 0.871864 & -0.748937 \\
\hline $\mathrm{C}$ & -2.359422 & 1.078502 & -0.291079 \\
\hline $\mathrm{N}$ & -2.083092 & 0.979147 & 1.111816 \\
\hline S & -2.035439 & -0.466965 & 1.866709 \\
\hline $\mathrm{O}$ & -3.262006 & -1.168129 & 1.559541 \\
\hline $\mathrm{O}$ & -1.664817 & -0.182265 & 3.234118 \\
\hline C & -0.724556 & -1.337306 & 1.09757 \\
\hline $\mathrm{C}$ & 0.571915 & -1.212108 & 1.592205 \\
\hline $\mathrm{C}$ & 1.613643 & -1.845813 & 0.954658 \\
\hline $\mathrm{C}$ & 1.364096 & -2.610842 & -0.180074 \\
\hline C & 0.073571 & -2.75516 & -0.664966 \\
\hline $\mathrm{C}$ & -0.974886 & -2.118692 & -0.015767 \\
\hline $\mathrm{H}$ & 2.314547 & 3.055766 & 2.259824 \\
\hline $\mathrm{H}$ & 4.742064 & 2.719252 & 1.894557 \\
\hline $\mathrm{H}$ & 5.557628 & 1.574756 & -0.109965 \\
\hline $\mathrm{H}$ & 3.993875 & 0.729379 & -1.818242 \\
\hline $\mathrm{H}$ & 1.114414 & 0.526819 & -2.265141 \\
\hline $\mathrm{H}$ & -0.833806 & 1.611599 & -3.256871 \\
\hline $\mathrm{H}$ & -3.11542 & 1.229986 & -4.061396 \\
\hline $\mathrm{H}$ & -4.924992 & 0.759638 & -2.452271 \\
\hline $\mathrm{H}$ & -4.423068 & 0.668683 & -0.037316 \\
\hline $\mathrm{H}$ & -1.451995 & 1.671431 & 1.487323 \\
\hline $\mathrm{H}$ & 0.752852 & -0.627921 & 2.473276 \\
\hline $\mathrm{H}$ & 2.618692 & -1.767853 & 1.315922 \\
\hline $\mathrm{H}$ & -0.130737 & -3.361115 & -1.523852 \\
\hline $\mathrm{H}$ & -1.979672 & -2.245422 & -0.369434 \\
\hline $\mathrm{O}$ & 2.466039 & -3.196004 & -0.76291 \\
\hline $\mathrm{C}$ & 2.337952 & -4.182103 & -1.814714 \\
\hline $\mathrm{H}$ & 3.343695 & -4.519264 & -2.002801 \\
\hline $\mathrm{H}$ & 1.720365 & -5.008763 & -1.486404 \\
\hline $\mathrm{H}$ & 1.921847 & -3.73615 & -2.709725 \\
\hline
\end{tabular}


Table S11: Cartesian atomic coordinates for compound $2 c\left(\mathrm{~S}_{0}, \mathrm{RHF} / 3-21+\mathrm{G}(\mathrm{d}, \mathrm{p})\right.$, $\mathrm{E}=-1657.8540427507$ a.u.)

\begin{tabular}{|c|c|c|c|}
\hline Atom & $X(\AA)$ & $Y(\AA)$ & $\mathrm{Z}(\AA)$ \\
\hline $\mathrm{C}$ & 2.004967 & -1.562663 & -1.554816 \\
\hline $\mathrm{C}$ & 3.113841 & -1.595534 & -2.388034 \\
\hline $\mathrm{C}$ & 4.297584 & -2.072863 & -1.861439 \\
\hline $\mathrm{C}$ & 4.374999 & -2.517238 & -0.53181 \\
\hline $\mathrm{C}$ & 3.273166 & -2.498603 & 0.301626 \\
\hline $\mathrm{C}$ & 2.091244 & -2.012447 & -0.236589 \\
\hline $\mathrm{N}$ & 0.814667 & -1.851797 & 0.289988 \\
\hline $\mathrm{N}$ & 0.693228 & -1.130846 & -1.807811 \\
\hline C & 0.025221 & -1.317238 & -0.704386 \\
\hline $\mathrm{C}$ & -1.395876 & -1.015228 & -0.500733 \\
\hline $\mathrm{C}$ & -2.152725 & -1.81846 & 0.334158 \\
\hline $\mathrm{C}$ & -3.498564 & -1.55384 & 0.533169 \\
\hline $\mathrm{C}$ & -4.095364 & -0.479004 & -0.100245 \\
\hline C & -3.338851 & 0.324946 & -0.94161 \\
\hline C & -1.998634 & 0.069036 & -1.148295 \\
\hline $\mathrm{N}$ & -1.224851 & 0.951982 & -1.971768 \\
\hline$S$ & -0.740479 & 2.40782 & -1.419178 \\
\hline $\mathrm{O}$ & -1.907913 & 3.102669 & -0.92221 \\
\hline $\mathrm{O}$ & 0.070029 & 2.977882 & -2.471817 \\
\hline C & 0.285765 & 2.060696 & -0.043028 \\
\hline C & 1.650375 & 1.853667 & -0.232285 \\
\hline $\mathrm{C}$ & 2.449188 & 1.522369 & 0.837986 \\
\hline C & 1.887292 & 1.397058 & 2.104389 \\
\hline C & 0.533896 & 1.62164 & 2.302228 \\
\hline $\mathrm{C}$ & -0.266407 & 1.962332 & 1.221001 \\
\hline $\mathrm{H}$ & 3.042231 & -1.260308 & -3.403974 \\
\hline $\mathrm{H}$ & 5.17557 & -2.112045 & -2.47659 \\
\hline $\mathrm{H}$ & 5.311216 & -2.884366 & -0.157413 \\
\hline $\mathrm{H}$ & 3.336005 & -2.8403 & 1.31672 \\
\hline $\mathrm{H}$ & 0.542869 & -1.992188 & 1.237766 \\
\hline $\mathrm{H}$ & -1.73286 & -2.681136 & 0.81049 \\
\hline $\mathrm{H}$ & -3.785295 & 1.165394 & -1.431942 \\
\hline $\mathrm{H}$ & 2.075372 & 1.96043 & -1.211183 \\
\hline $\mathrm{H}$ & 3.501109 & 1.360471 & 0.719788 \\
\hline $\mathrm{H}$ & 0.097142 & 1.549068 & 3.277208 \\
\hline $\mathrm{H}$ & -1.309737 & 2.162824 & 1.368702 \\
\hline $\mathrm{O}$ & 2.75566 & 1.043618 & 3.113314 \\
\hline $\mathrm{C}$ & 2.36008 & 1.077698 & 4.505259 \\
\hline $\mathrm{H}$ & 3.257126 & 0.844191 & 5.054261 \\
\hline $\mathrm{H}$ & 2.001855 & 2.063686 & 4.773799 \\
\hline $\mathrm{H}$ & 1.597748 & 0.334855 & 4.705374 \\
\hline $\mathrm{H}$ & -5.133473 & -0.259772 & 0.040553 \\
\hline $\mathrm{O}$ & -4.158613 & -2.42022 & 1.378462 \\
\hline C & -5.575984 & -2.287608 & 1.638689 \\
\hline $\mathrm{H}$ & -5.813023 & -3.099769 & 2.305737 \\
\hline $\mathrm{H}$ & -5.78837 & -1.338689 & 2.115549 \\
\hline $\mathrm{H}$ & -6.141521 & -2.381849 & 0.719983 \\
\hline $\mathrm{H}$ & -0.59221 & 0.515122 & -2.624252 \\
\hline
\end{tabular}


Table S12: Cartesian atomic coordinates for compound 3c ( $\mathrm{S}_{0}, \mathrm{RHF} / 3-21+\mathrm{G}(\mathrm{d}, \mathrm{p})$, $E=-1657.8559021637$ a.u.)

\begin{tabular}{|c|c|c|c|}
\hline Atom & $X(\AA)$ & $Y(\AA)$ & $\mathrm{Z}(\AA)$ \\
\hline $\mathrm{C}$ & 2.28953 & 2.069081 & 0.314205 \\
\hline C & 3.289453 & 2.544326 & 1.149596 \\
\hline C & 4.596299 & 2.427484 & 0.71717 \\
\hline C & 4.90187 & 1.855856 & -0.527258 \\
\hline $\mathrm{C}$ & 3.910304 & 1.39269 & -1.371993 \\
\hline C & 2.603566 & 1.511234 & -0.925629 \\
\hline $\mathrm{N}$ & 1.380342 & 1.167816 & -1.493794 \\
\hline $\mathrm{N}$ & 0.896086 & 2.053385 & 0.484665 \\
\hline $\mathrm{C}$ & 0.394965 & 1.520399 & -0.596205 \\
\hline $\mathrm{C}$ & -1.025497 & 1.285603 & -0.856583 \\
\hline $\mathrm{C}$ & -1.526849 & 1.335491 & -2.15859 \\
\hline $\mathrm{C}$ & -2.854947 & 1.091577 & -2.422367 \\
\hline $\mathrm{C}$ & -3.716929 & 0.78688 & -1.375138 \\
\hline $\mathrm{C}$ & -3.246744 & 0.745815 & -0.076262 \\
\hline $\mathrm{C}$ & -1.908017 & 0.996203 & 0.181091 \\
\hline $\mathrm{N}$ & -1.432227 & 0.887488 & 1.527697 \\
\hline S & -1.264225 & -0.570863 & 2.245794 \\
\hline $\mathrm{O}$ & -2.525155 & -1.270327 & 2.128832 \\
\hline $\mathrm{O}$ & -0.683152 & -0.305399 & 3.541562 \\
\hline C & -0.091761 & -1.418737 & 1.260316 \\
\hline C & 1.267236 & -1.286736 & 1.537842 \\
\hline $\mathrm{C}$ & 2.195752 & -1.888989 & 0.720532 \\
\hline C & 1.769815 & -2.630593 & -0.37696 \\
\hline C & 0.418478 & -2.78309 & -0.645338 \\
\hline $\mathrm{C}$ & -0.514422 & -2.177081 & 0.183894 \\
\hline $\mathrm{H}$ & 3.045206 & 2.985904 & 2.095637 \\
\hline $\mathrm{H}$ & 5.395277 & 2.783422 & 1.338311 \\
\hline $\mathrm{H}$ & 5.928264 & 1.783009 & -0.831538 \\
\hline $\mathrm{H}$ & 4.147664 & 0.95973 & -2.324598 \\
\hline $\mathrm{H}$ & 1.245603 & 0.653919 & -2.335885 \\
\hline $\mathrm{H}$ & -0.873996 & 1.596941 & -2.969391 \\
\hline $\mathrm{H}$ & -3.245869 & 1.136475 & -3.417873 \\
\hline $\mathrm{H}$ & -3.8861 & 0.514347 & 0.747201 \\
\hline $\mathrm{H}$ & -0.733972 & 1.567125 & 1.795727 \\
\hline $\mathrm{H}$ & 1.585206 & -0.718837 & 2.390287 \\
\hline $\mathrm{H}$ & 3.245922 & -1.802955 & 0.911387 \\
\hline $\mathrm{H}$ & 0.081203 & -3.369471 & -1.475288 \\
\hline $\mathrm{H}$ & -1.562344 & -2.305803 & -0.005319 \\
\hline $\mathrm{O}$ & 2.766163 & -3.18344 & -1.149339 \\
\hline C & 2.475855 & -4.129579 & -2.205581 \\
\hline $\mathrm{H}$ & 3.440106 & -4.442963 & -2.569634 \\
\hline $\mathrm{H}$ & 1.928548 & -4.978298 & -1.814884 \\
\hline $\mathrm{H}$ & 1.913984 & -3.655363 & -3.000825 \\
\hline $\mathrm{O}$ & -5.025484 & 0.54451 & -1.714932 \\
\hline C & -6.023238 & 0.235408 & -0.708562 \\
\hline $\mathrm{H}$ & -6.135646 & 1.064365 & -0.021514 \\
\hline $\mathrm{H}$ & -6.934521 & 0.083126 & -1.262159 \\
\hline $\mathrm{H}$ & -5.753652 & -0.663907 & -0.170387 \\
\hline
\end{tabular}


Table S13: Cartesian atomic coordinates for the phototautomer of $1 \mathrm{c}$ $\left(\mathrm{S}_{0}, \mathrm{RHF} / 3-21+\mathrm{G}(\mathrm{d}, \mathrm{p}), \mathrm{E}=-1544.5630606722\right.$ a.u.)

\begin{tabular}{|c|c|c|c|}
\hline Atom & $\mathrm{X}(\AA)$ & $\mathrm{Y}(\AA)$ & $Z(\AA)$ \\
\hline $\mathrm{C}$ & 3.583876 & 1.056592 & -0.040366 \\
\hline $\mathrm{C}$ & 4.024964 & 2.274726 & -0.520857 \\
\hline $\mathrm{C}$ & 5.284979 & 2.687698 & -0.12105 \\
\hline $\mathrm{C}$ & 6.071923 & 1.905194 & 0.7296 \\
\hline $\mathrm{C}$ & 5.625174 & 0.683256 & 1.206935 \\
\hline $\mathrm{C}$ & 4.368236 & 0.278671 & 0.802438 \\
\hline $\mathrm{N}$ & 3.613538 & -0.873452 & 1.0772 \\
\hline $\mathrm{N}$ & 2.397132 & 0.352307 & -0.236995 \\
\hline $\mathrm{C}$ & 2.421236 & -0.80057 & 0.427587 \\
\hline C & 1.367273 & -1.775869 & 0.437436 \\
\hline C & 1.517063 & -2.965439 & 1.175093 \\
\hline C & 0.541036 & -3.919202 & 1.184734 \\
\hline $\mathrm{C}$ & -0.623824 & -3.700667 & 0.428581 \\
\hline C & -0.803536 & -2.556554 & -0.295753 \\
\hline $\mathrm{C}$ & 0.185479 & -1.539611 & -0.316318 \\
\hline $\mathrm{N}$ & 0.095563 & -0.36206 & -0.991807 \\
\hline$S$ & -1.079258 & 0.156821 & -1.921187 \\
\hline $\mathrm{O}$ & -1.539484 & -0.843423 & -2.878214 \\
\hline $\mathrm{O}$ & -0.625793 & 1.436963 & -2.446039 \\
\hline $\mathrm{C}$ & -2.443893 & 0.455614 & -0.845142 \\
\hline $\mathrm{C}$ & -2.284523 & 1.280525 & 0.254371 \\
\hline $\mathrm{C}$ & -3.349751 & 1.532348 & 1.103325 \\
\hline $\mathrm{C}$ & -4.583783 & 0.95613 & 0.838327 \\
\hline $\mathrm{C}$ & -4.751898 & 0.137802 & -0.269638 \\
\hline C & -3.685257 & -0.109025 & -1.110375 \\
\hline $\mathrm{H}$ & 3.416425 & 2.866126 & -1.175544 \\
\hline $\mathrm{H}$ & 5.666779 & 3.626032 & -0.471786 \\
\hline $\mathrm{H}$ & 7.042838 & 2.257462 & 1.01737 \\
\hline $\mathrm{H}$ & 6.230888 & 0.083167 & 1.857082 \\
\hline $\mathrm{H}$ & 3.909672 & -1.630158 & 1.650719 \\
\hline $\mathrm{H}$ & 2.411637 & -3.137131 & 1.743956 \\
\hline $\mathrm{H}$ & 0.661645 & -4.822729 & 1.748003 \\
\hline $\mathrm{H}$ & -1.684435 & -2.433686 & -0.887595 \\
\hline $\mathrm{H}$ & 1.545636 & 0.538856 & -0.790833 \\
\hline $\mathrm{H}$ & -1.330156 & 1.729357 & 0.452465 \\
\hline $\mathrm{H}$ & -3.209147 & 2.171698 & 1.950361 \\
\hline $\mathrm{H}$ & -5.717235 & -0.286537 & -0.45527 \\
\hline $\mathrm{H}$ & -3.806037 & -0.733589 & -1.973016 \\
\hline $\mathrm{O}$ & -5.698826 & 1.146613 & 1.62434 \\
\hline $\mathrm{C}$ & -5.672737 & 2.008502 & 2.784024 \\
\hline $\mathrm{H}$ & -5.417027 & 3.021972 & 2.500595 \\
\hline $\mathrm{H}$ & -4.970899 & 1.637126 & 3.520575 \\
\hline $\mathrm{H}$ & -6.674976 & 1.975341 & 3.178006 \\
\hline $\mathrm{H}$ & -1.390512 & -4.45197 & 0.416716 \\
\hline
\end{tabular}


Table S14: Cartesian atomic coordinates for the phototautomer of compound 2c $\left(\mathrm{S}_{0}, \mathrm{RHF} / 3-21+\mathrm{G}(\mathrm{d}, \mathrm{p}), \mathrm{E}=-1657.8301403267\right.$ a.u. $)$

\begin{tabular}{|c|c|c|c|}
\hline Atom & X (§) & $Y(\AA)$ & $\mathrm{Z}(\AA)$ \\
\hline $\mathrm{C}$ & 3.359543 & 1.748996 & 0.17416 \\
\hline C & 3.690609 & 3.08513 & 0.055055 \\
\hline $\mathrm{C}$ & 4.928296 & 3.471058 & 0.542665 \\
\hline C & 5.800484 & 2.54814 & 1.127559 \\
\hline $\mathrm{C}$ & 5.463971 & 1.208487 & 1.242774 \\
\hline $\mathrm{C}$ & 4.228747 & 0.832364 & 0.753298 \\
\hline $\mathrm{N}$ & 3.573393 & -0.40881 & 0.697934 \\
\hline $\mathrm{N}$ & 2.223413 & 1.034506 & -0.201438 \\
\hline C & 2.356705 & -0.25425 & 0.108256 \\
\hline C & 1.381719 & -1.272331 & -0.148893 \\
\hline C & 1.655483 & -2.61515 & 0.223465 \\
\hline $\mathrm{C}$ & 0.752914 & -3.594777 & -0.032402 \\
\hline $\mathrm{C}$ & -0.454292 & -3.27248 & -0.68129 \\
\hline $\mathrm{C}$ & -0.739549 & -1.995189 & -1.045247 \\
\hline $\mathrm{C}$ & 0.17124 & -0.929311 & -0.788225 \\
\hline $\mathrm{N}$ & -0.039574 & 0.380187 & -1.105122 \\
\hline S & -1.284157 & 1.024636 & -1.843717 \\
\hline $\mathrm{O}$ & -1.689876 & 0.285561 & -3.034789 \\
\hline $\mathrm{O}$ & -0.962755 & 2.435809 & -2.001021 \\
\hline $\mathrm{C}$ & -2.633349 & 0.889323 & -0.715796 \\
\hline $\mathrm{C}$ & -2.501372 & 1.368188 & 0.57573 \\
\hline $\mathrm{C}$ & -3.556158 & 1.27814 & 1.469231 \\
\hline $\mathrm{C}$ & -4.752518 & 0.709343 & 1.05665 \\
\hline $\mathrm{C}$ & -4.893815 & 0.237779 & -0.240751 \\
\hline $\mathrm{C}$ & -3.837827 & 0.330369 & -1.124896 \\
\hline $\mathrm{H}$ & 3.017384 & 3.785164 & -0.398136 \\
\hline $\mathrm{H}$ & 5.225419 & 4.498433 & 0.468953 \\
\hline $\mathrm{H}$ & 6.750841 & 2.883594 & 1.493424 \\
\hline $\mathrm{H}$ & 6.135076 & 0.501476 & 1.689433 \\
\hline $\mathrm{H}$ & 3.946247 & -1.268523 & 1.030158 \\
\hline $\mathrm{H}$ & 2.579389 & -2.855901 & 0.708826 \\
\hline $\mathrm{H}$ & -1.142235 & -4.068431 & -0.884064 \\
\hline $\mathrm{H}$ & -1.648059 & -1.785513 & -1.566586 \\
\hline $\mathrm{H}$ & 1.34183 & 1.299122 & -0.670945 \\
\hline $\mathrm{H}$ & -1.576098 & 1.811973 & 0.889563 \\
\hline $\mathrm{H}$ & -3.436846 & 1.652 & 2.465223 \\
\hline $\mathrm{H}$ & -5.830606 & -0.189251 & -0.534787 \\
\hline $\mathrm{H}$ & -3.938485 & -0.025313 & -2.131004 \\
\hline $\mathrm{O}$ & 0.910282 & -4.93437 & 0.28723 \\
\hline $\mathrm{C}$ & 2.109786 & -5.410165 & 0.927674 \\
\hline $\mathrm{H}$ & 2.977196 & -5.222937 & 0.30448 \\
\hline $\mathrm{H}$ & 1.965485 & -6.471326 & 1.04753 \\
\hline $\mathrm{H}$ & 2.238144 & -4.942732 & 1.897685 \\
\hline $\mathrm{O}$ & -5.85441 & 0.575316 & 1.872235 \\
\hline $\mathrm{C}$ & -5.856121 & 1.061329 & 3.233132 \\
\hline $\mathrm{H}$ & -5.683038 & 2.130136 & 3.25689 \\
\hline $\mathrm{H}$ & -5.107558 & 0.547391 & 3.823504 \\
\hline $\mathrm{H}$ & -6.841097 & 0.836787 & 3.607573 \\
\hline
\end{tabular}


Table S15: Cartesian atomic coordinates for the phototautomer of compound 3c $\left(\mathrm{S}_{0}, \mathrm{RHF} / 3-21+\mathrm{G}(\mathrm{d}, \mathrm{p}), \mathrm{E}=-1657.837067599\right.$ a.u. $)$

\begin{tabular}{|c|c|c|c|}
\hline Atom & $X(\AA)$ & $\mathrm{Y}(\AA)$ & $\mathrm{Z}(\AA)$ \\
\hline $\mathrm{C}$ & 3.864814 & 1.04865 & 0.072226 \\
\hline $\mathrm{C}$ & 4.451684 & 2.251658 & -0.268954 \\
\hline $\mathrm{C}$ & 5.744212 & 2.469377 & 0.180102 \\
\hline $\mathrm{C}$ & 6.420425 & 1.513377 & 0.943146 \\
\hline $\mathrm{C}$ & 5.826714 & 0.307049 & 1.281445 \\
\hline $\mathrm{C}$ & 4.539416 & 0.098147 & 0.828907 \\
\hline $\mathrm{N}$ & 3.651848 & -0.981444 & 0.972876 \\
\hline $\mathrm{N}$ & 2.608153 & 0.513143 & -0.206171 \\
\hline C & 2.485859 & -0.700092 & 0.329058 \\
\hline $\mathrm{C}$ & 1.329435 & -1.537375 & 0.227195 \\
\hline C & 1.315925 & -2.801828 & 0.83131 \\
\hline $\mathrm{C}$ & 0.235667 & -3.633873 & 0.740977 \\
\hline $\mathrm{C}$ & -0.881791 & -3.198536 & 0.006375 \\
\hline $\mathrm{C}$ & -0.905278 & -1.969834 & -0.60235 \\
\hline $\mathrm{C}$ & 0.186578 & -1.090676 & -0.508001 \\
\hline $\mathrm{N}$ & 0.250374 & 0.155325 & -1.049035 \\
\hline S & -0.847698 & 0.908018 & -1.912145 \\
\hline $\mathrm{O}$ & -1.38073 & 0.095784 & -2.999441 \\
\hline $\mathrm{O}$ & -0.257719 & 2.194247 & -2.256604 \\
\hline $\mathrm{C}$ & -2.198472 & 1.195167 & -0.815743 \\
\hline $\mathrm{C}$ & -1.985297 & 1.868388 & 0.374129 \\
\hline C & -3.036848 & 2.10599 & 1.243984 \\
\hline $\mathrm{C}$ & -4.310336 & 1.668667 & 0.909087 \\
\hline C & -4.531124 & 1.002378 & -0.287617 \\
\hline C & -3.477849 & 0.768601 & -1.148696 \\
\hline $\mathrm{H}$ & 3.92697 & 2.977739 & -0.857275 \\
\hline $\mathrm{H}$ & 6.237343 & 3.389527 & -0.063639 \\
\hline $\mathrm{H}$ & 7.420259 & 1.716494 & 1.272782 \\
\hline $\mathrm{H}$ & 6.347629 & -0.42627 & 1.865132 \\
\hline $\mathrm{H}$ & 3.850323 & -1.824519 & 1.461376 \\
\hline $\mathrm{H}$ & 2.169355 & -3.143841 & 1.386555 \\
\hline $\mathrm{H}$ & 0.250654 & -4.592299 & 1.213977 \\
\hline $\mathrm{H}$ & -1.764515 & -1.709179 & -1.178195 \\
\hline $\mathrm{H}$ & 1.793747 & 0.8568 & -0.739665 \\
\hline $\mathrm{H}$ & -0.999796 & 2.210466 & 0.626014 \\
\hline $\mathrm{H}$ & -2.855158 & 2.627852 & 2.160894 \\
\hline $\mathrm{H}$ & -5.524882 & 0.681124 & -0.523487 \\
\hline $\mathrm{H}$ & -3.638043 & 0.258632 & -2.077847 \\
\hline $\mathrm{O}$ & -2.005122 & -3.966196 & -0.145132 \\
\hline C & -2.115785 & -5.301554 & 0.400546 \\
\hline $\mathrm{H}$ & -2.046138 & -5.279838 & 1.480902 \\
\hline $\mathrm{H}$ & -1.352828 & -5.947518 & -0.015441 \\
\hline $\mathrm{H}$ & -3.093099 & -5.640109 & 0.099341 \\
\hline $\mathrm{O}$ & -5.416063 & 1.857426 & 1.709864 \\
\hline C & -5.333056 & 2.574044 & 2.961569 \\
\hline $\mathrm{H}$ & -4.991503 & 3.588837 & 2.798761 \\
\hline $\mathrm{H}$ & -4.672871 & 2.063236 & 3.65178 \\
\hline $\mathrm{H}$ & -6.339213 & 2.578984 & 3.347012 \\
\hline
\end{tabular}


Table S16: Cartesian atomic coordinates for the phototautomer of compound 1c $\left(\mathrm{S}_{1}, \mathrm{RHF}-\mathrm{CIS} / 3-21+\mathrm{G}(\mathrm{d}, \mathrm{p}), \mathrm{E}=-1544.5512376899\right.$ a.u. $)$

\begin{tabular}{|c|c|c|c|}
\hline Atom & $X(\AA)$ & $\mathrm{Y}(\AA)$ & $\mathrm{Z}(\AA)$ \\
\hline $\mathrm{C}$ & 3.392772 & 1.082939 & -0.328522 \\
\hline $\mathrm{C}$ & 3.824998 & 2.173135 & -1.060589 \\
\hline $\mathrm{C}$ & 5.021402 & 2.766257 & -0.674822 \\
\hline C & 5.75508 & 2.283308 & 0.407097 \\
\hline $\mathrm{C}$ & 5.318432 & 1.185216 & 1.142561 \\
\hline $\mathrm{C}$ & 4.133 & 0.59707 & 0.755756 \\
\hline $\mathrm{N}$ & 3.426896 & -0.494594 & 1.25417 \\
\hline $\mathrm{N}$ & 2.277014 & 0.280205 & -0.443792 \\
\hline $\mathrm{C}$ & 2.273245 & -0.701161 & 0.511854 \\
\hline $\mathrm{C}$ & 1.343282 & -1.728179 & 0.728869 \\
\hline $\mathrm{C}$ & 1.522864 & -2.674458 & 1.745706 \\
\hline $\mathrm{C}$ & 0.628975 & -3.727897 & 1.925843 \\
\hline $\mathrm{C}$ & -0.482039 & -3.887897 & 1.095194 \\
\hline C & -0.726214 & -2.965295 & 0.088051 \\
\hline $\mathrm{C}$ & 0.124147 & -1.862669 & -0.117873 \\
\hline $\mathrm{N}$ & -0.031471 & -0.879929 & -1.001013 \\
\hline S & -1.268646 & -0.561381 & -1.987098 \\
\hline $\mathrm{O}$ & -1.881625 & -1.770787 & -2.51207 \\
\hline $\mathrm{O}$ & -0.771147 & 0.433652 & -2.917689 \\
\hline C & -2.426187 & 0.205681 & -0.921751 \\
\hline C & -2.223464 & 1.518552 & -0.528247 \\
\hline C & -3.092837 & 2.120354 & 0.365102 \\
\hline C & -4.168926 & 1.399028 & 0.863117 \\
\hline $\mathrm{C}$ & -4.384053 & 0.087104 & 0.457773 \\
\hline $\mathrm{C}$ & -3.51706 & -0.506944 & -0.433306 \\
\hline $\mathrm{H}$ & 3.259999 & 2.540145 & -1.894498 \\
\hline $\mathrm{H}$ & 5.387299 & 3.612483 & -1.222605 \\
\hline $\mathrm{H}$ & 6.674118 & 2.76458 & 0.678497 \\
\hline $\mathrm{H}$ & 5.884813 & 0.813109 & 1.973801 \\
\hline $\mathrm{H}$ & 3.711628 & -1.048558 & 2.028525 \\
\hline $\mathrm{H}$ & 2.363325 & -2.611088 & 2.408342 \\
\hline $\mathrm{H}$ & 0.812084 & -4.433765 & 2.712613 \\
\hline $\mathrm{H}$ & -1.558662 & -3.096424 & -0.569826 \\
\hline $\mathrm{H}$ & 1.522153 & 0.324376 & -1.106365 \\
\hline $\mathrm{H}$ & -1.397974 & 2.073989 & -0.928526 \\
\hline $\mathrm{H}$ & -2.927423 & 3.136832 & 0.656649 \\
\hline $\mathrm{H}$ & -5.233538 & -0.437807 & 0.843332 \\
\hline $\mathrm{H}$ & -3.691511 & -1.509579 & -0.768022 \\
\hline $\mathrm{O}$ & -5.081108 & 1.90984 & 1.753711 \\
\hline $\mathrm{C}$ & -5.011137 & 3.277217 & 2.221011 \\
\hline $\mathrm{H}$ & -5.092515 & 3.967331 & 1.39065 \\
\hline $\mathrm{H}$ & -4.089495 & 3.44789 & 2.763074 \\
\hline $\mathrm{H}$ & -5.855617 & 3.387983 & 2.880456 \\
\hline $\mathrm{H}$ & -1.144069 & -4.718985 & 1.233349 \\
\hline
\end{tabular}


Table S17: Cartesian atomic coordinates for the phototautomer of compound 2c $\left(\mathrm{S}_{1}, \mathrm{RHF}-\mathrm{CIS} / 3-21+\mathrm{G}(\mathrm{d}, \mathrm{p}), \mathrm{E}=-1657.8180450134\right.$ a.u. $)$

\begin{tabular}{|c|c|c|c|}
\hline Atom & $X(\AA)$ & $\mathrm{Y}(\AA)$ & $\mathrm{Z}(\AA)$ \\
\hline $\mathrm{C}$ & 3.082047 & 1.94272 & -0.077324 \\
\hline $\mathrm{C}$ & 3.343205 & 3.270615 & -0.359221 \\
\hline $\mathrm{C}$ & 4.493191 & 3.824806 & 0.190999 \\
\hline $\mathrm{C}$ & 5.347567 & 3.073643 & 0.995349 \\
\hline C & 5.082749 & 1.736337 & 1.277771 \\
\hline $\mathrm{C}$ & 3.943115 & 1.188954 & 0.728452 \\
\hline $\mathrm{N}$ & 3.392833 & -0.089605 & 0.799726 \\
\hline $\mathrm{N}$ & 2.053179 & 1.106073 & -0.4569 \\
\hline C & 2.219548 & -0.148704 & 0.062072 \\
\hline $\mathrm{C}$ & 1.412204 & -1.285764 & -0.103766 \\
\hline $\mathrm{C}$ & 1.758314 & -2.508486 & 0.48479 \\
\hline $\mathrm{C}$ & 0.984285 & -3.648981 & 0.278606 \\
\hline $\mathrm{C}$ & -0.15787 & -3.619756 & -0.528794 \\
\hline C & -0.550857 & -2.428555 & -1.105484 \\
\hline C & 0.163299 & -1.232964 & -0.905766 \\
\hline $\mathrm{N}$ & -0.150539 & -0.016736 & -1.356856 \\
\hline S & -1.474777 & 0.481143 & -2.123212 \\
\hline $\mathrm{O}$ & -1.976601 & -0.511849 & -3.060827 \\
\hline $\mathrm{O}$ & -1.162765 & 1.810939 & -2.613524 \\
\hline C & -2.652368 & 0.632502 & -0.834449 \\
\hline C & -2.578173 & 1.711474 & 0.031163 \\
\hline $\mathrm{C}$ & -3.460959 & 1.816685 & 1.092265 \\
\hline C & -4.422049 & 0.833607 & 1.281726 \\
\hline $\mathrm{C}$ & -4.509543 & -0.241502 & 0.406019 \\
\hline $\mathrm{C}$ & -3.629341 & -0.340611 & -0.650083 \\
\hline $\mathrm{H}$ & 2.685521 & 3.844859 & -0.981267 \\
\hline $\mathrm{H}$ & 4.727399 & 4.851906 & -0.009506 \\
\hline $\mathrm{H}$ & 6.226526 & 3.53204 & 1.40429 \\
\hline $\mathrm{H}$ & 5.742131 & 1.158774 & 1.89582 \\
\hline $\mathrm{H}$ & 3.79006 & -0.848276 & 1.303145 \\
\hline $\mathrm{H}$ & 2.629936 & -2.583915 & 1.099932 \\
\hline $\mathrm{H}$ & -0.704641 & -4.526029 & -0.681681 \\
\hline $\mathrm{H}$ & -1.407018 & -2.40924 & -1.745406 \\
\hline $\mathrm{H}$ & 1.248701 & 1.291158 & -1.034319 \\
\hline $\mathrm{H}$ & -1.842745 & 2.475206 & -0.130824 \\
\hline $\mathrm{H}$ & -3.395286 & 2.658579 & 1.749944 \\
\hline $\mathrm{H}$ & -5.273282 & -0.974406 & 0.565568 \\
\hline $\mathrm{H}$ & -3.708635 & -1.153688 & -1.343155 \\
\hline $\mathrm{O}$ & 1.286791 & -4.862248 & 0.829645 \\
\hline $\mathrm{C}$ & 2.442339 & -5.082419 & 1.671571 \\
\hline $\mathrm{H}$ & 3.354754 & -4.875986 & 1.126294 \\
\hline $\mathrm{H}$ & 2.395995 & -6.125809 & 1.934609 \\
\hline $\mathrm{H}$ & 2.38538 & -4.470612 & 2.563044 \\
\hline $\mathrm{O}$ & -5.338016 & 0.851409 & 2.306091 \\
\hline C & -5.396984 & 1.93778 & 3.259133 \\
\hline $\mathrm{H}$ & -5.598366 & 2.875036 & 2.755676 \\
\hline $\mathrm{H}$ & -4.473123 & 2.003472 & 3.820106 \\
\hline $\mathrm{H}$ & -6.212801 & 1.686218 & 3.916072 \\
\hline
\end{tabular}


Table S18: Cartesian atomic coordinates for the phototautomer of compound 3c $\left(\mathrm{S}_{1}, \mathrm{RHF}-\mathrm{CIS} / 3-21+\mathrm{G}(\mathrm{d}, \mathrm{p}), \mathrm{E}=-1657.8257548909\right.$ a.u. $)$

\begin{tabular}{|c|c|c|c|}
\hline Atom & $X(\AA)$ & $Y(\AA)$ & $\mathrm{Z}(\AA)$ \\
\hline $\mathrm{C}$ & 3.76467 & 1.051928 & -0.092756 \\
\hline C & 4.391819 & 2.190802 & -0.564778 \\
\hline $\mathrm{C}$ & 5.652998 & 2.480578 & -0.057554 \\
\hline C & 6.261855 & 1.658537 & 0.889167 \\
\hline $\mathrm{C}$ & 5.629683 & 0.512296 & 1.363015 \\
\hline C & 4.379868 & 0.224778 & 0.856501 \\
\hline $\mathrm{N}$ & 3.491924 & -0.817859 & 1.101711 \\
\hline $\mathrm{N}$ & 2.541338 & 0.48919 & -0.383323 \\
\hline $\mathrm{C}$ & 2.345357 & -0.665507 & 0.331779 \\
\hline $\mathrm{C}$ & 1.257749 & -1.547283 & 0.323732 \\
\hline $\mathrm{C}$ & 1.236852 & -2.69918 & 1.12071 \\
\hline $\mathrm{C}$ & 0.173564 & -3.604332 & 1.07593 \\
\hline $\mathrm{C}$ & -0.90757 & -3.391147 & 0.225678 \\
\hline $\mathrm{C}$ & -0.956291 & -2.261115 & -0.574541 \\
\hline $\mathrm{C}$ & 0.063409 & -1.300503 & -0.537087 \\
\hline $\mathrm{N}$ & 0.101081 & -0.144693 & -1.190244 \\
\hline S & -1.033764 & 0.550003 & -2.107702 \\
\hline $\mathrm{O}$ & -1.714925 & -0.412065 & -2.957447 \\
\hline $\mathrm{O}$ & -0.378148 & 1.688122 & -2.723306 \\
\hline C & -2.191547 & 1.137941 & -0.933071 \\
\hline C & -1.874149 & 2.241514 & -0.157847 \\
\hline C & -2.753311 & 2.685739 & 0.814511 \\
\hline C & -3.956229 & 2.019306 & 1.003905 \\
\hline $\mathrm{C}$ & -4.284024 & 0.922155 & 0.217012 \\
\hline $\mathrm{C}$ & -3.405254 & 0.483283 & -0.749908 \\
\hline $\mathrm{H}$ & 3.922321 & 2.819457 & -1.295231 \\
\hline $\mathrm{H}$ & 6.168488 & 3.354984 & -0.4036 \\
\hline $\mathrm{H}$ & 7.235766 & 1.91181 & 1.259683 \\
\hline $\mathrm{H}$ & 6.099658 & -0.120369 & 2.090393 \\
\hline $\mathrm{H}$ & 3.66016 & -1.570264 & 1.728702 \\
\hline $\mathrm{H}$ & 2.045887 & -2.918588 & 1.788529 \\
\hline $\mathrm{H}$ & 0.212814 & -4.470377 & 1.703904 \\
\hline $\mathrm{H}$ & -1.779973 & -2.141765 & -1.242974 \\
\hline $\mathrm{H}$ & 1.82862 & 0.801782 & -1.019394 \\
\hline $\mathrm{H}$ & -0.949244 & 2.760232 & -0.319806 \\
\hline $\mathrm{H}$ & -2.497578 & 3.541674 & 1.404007 \\
\hline $\mathrm{H}$ & -5.225703 & 0.438051 & 0.374356 \\
\hline $\mathrm{H}$ & -3.661517 & -0.349381 & -1.373734 \\
\hline $\mathrm{O}$ & -1.984745 & -4.24996 & 0.118599 \\
\hline C & -2.038272 & -5.493278 & 0.850351 \\
\hline $\mathrm{H}$ & -2.046738 & -5.310259 & 1.91853 \\
\hline $\mathrm{H}$ & -1.202444 & -6.13001 & 0.584782 \\
\hline $\mathrm{H}$ & -2.964213 & -5.954675 & 0.548852 \\
\hline $\mathrm{O}$ & -4.888906 & 2.385558 & 1.943842 \\
\hline C & -4.703776 & 3.540104 & 2.795329 \\
\hline $\mathrm{H}$ & -4.611506 & 4.440376 & 2.200811 \\
\hline $\mathrm{H}$ & -3.831476 & 3.413949 & 3.424352 \\
\hline $\mathrm{H}$ & -5.594122 & 3.583899 & 3.400087 \\
\hline
\end{tabular}


Summary of Structural Parameters for the Optimized Geometries (Table S19-S21)

All interatomic distances are reported in Angstroms $(\AA)$; dihedral angles and bond angles are reported in degrees (deg). The corresponding level of theory is indicated for each molecule. The numbering scheme is included above the data tables. "PT" is used to indicate the imino tautomer form of the compound. "Para" indicates substitution at the 5- position and "meta" indicates substitution at the 4- position (numbering scheme in Chart 1 ). " $S_{0}$ " corresponds to a ground state calculation and " $S_{1}$ " corresponds to the first excited singlet state.

Table S19: Selected interatomic distances ( $\AA$ ) for geometry-optimized structures.

\begin{tabular}{lccccccccc}
\hline & C2-C7 & C7=N1 & C7-N2 & C1-N3 & N3-S1 & H1--N & 7 C-R & S1-C14 & C1=C2 \\
\hline 1c & & & & & & & & & \\
Exp. (X-ray) & 1.4647 & 1.3239 & 1.3619 & 1.4442 & 1.6474 & 1.8178 & $* * *$ & 1.7510 & 1.3974 \\
B3LYP/6-31G* & 1.4651 & 1.3235 & 1.3829 & 1.4081 & 1.6949 & 1.8647 & $* * *$ & 1.7949 & 1.4238 \\
HF/3-21+G** & 1.4665 & 1.3036 & 1.3790 & 1.4331 & 1.6315 & 2.2047 & $* * *$ & 1.7551 & 1.3964 \\
HF/6-31+G** & 1.4778 & 1.2910 & 1.3647 & 1.4146 & 1.6459 & 2.0714 & $* * *$ & 1.7700 & 1.4020 \\
PT-1c & & & & & & & & & \\
B3LYP/6-31G* & 1.4326 & 1.3477 & 1.3694 & 1.3561 & 1.6482 & 1.6719 & $* * *$ & 1.8041 & 1.4504 \\
HF/3-21+G** & 1.4361 & 1.3309 & 1.3597 & 1.3604 & 1.5847 & 1.7203 & $* * *$ & 1.7656 & 1.4215 \\
HF-CIS/3-21+G** & 1.4025 & 1.3699 & 1.3873 & 1.3297 & 1.6131 & 1.9664 & $* * *$ & 1.7553 & 1.4908 \\
2c & & & & & & & & & \\
B3LYP/6-31G* & 1.4651 & 1.3227 & 1.3838 & 1.4159 & 1.6980 & 1.9116 & 1.3650 & 1.7969 & 1.4152 \\
HF/3-21+G** & 1.4672 & 1.3024 & 1.3798 & 1.4337 & 1.6297 & 2.2673 & 1.3783 & 1.7553 & 1.3881 \\
PT-2c & & & & & & & & & \\
B3LYP/6-31G* & 1.4291 & 1.3496 & 1.3708 & 1.3583 & 1.3455 & 1.6777 & 1.3700 & 1.8055 & 1.4439 \\
HF/3-21+G** & 1.4328 & 1.3321 & 1.3609 & 1.3633 & 1.5836 & 1.7180 & 1.3863 & 1.7665 & 1.4113 \\
HF-CIS/3-21+G** & 1.4045 & 1.3679 & 1.3871 & 1.3341 & 1.6083 & 1.9416 & 1.3664 & 1.7569 & 1.4852 \\
3c & & & & & & & & & \\
B3LYP/6-31G* & 1.4618 & 1.3243 & 1.3840 & 1.4040 & 1.6893 & 1.8417 & 1.3600 & 1.7955 & 1.4296 \\
HF/3-21+G** & 1.4639 & 1.3041 & 1.3798 & 1.4319 & 1.6321 & 2.1916 & 1.3739 & 1.7553 & 1.4033 \\
PT-3c & & & & & & & & & \\
B3LYP/6-31G* & 1.4270 & 1.3500 & 1.3699 & 1.3568 & 1.6479 & 1.7116 & 1.3605 & 1.8038 & 1.4547 \\
HF/3-21+G** & 1.4315 & 1.3316 & 1.3613 & 1.3598 & 1.5861 & 1.7245 & 1.3695 & 1.7659 & 1.4303 \\
HF-CIS/3-21+G** & 1.4002 & 1.3722 & 1.3895 & 1.3278 & 1.6155 & 1.9761 & 1.3821 & 1.7549 & 1.4930 \\
\hline
\end{tabular}


Table S20: Selected bond angles (deg) for geometry-optimized structures.

\begin{tabular}{|c|c|c|c|c|c|c|c|c|}
\hline & C14-S1-N3 & S1-N3-C1 & $\mathrm{N} 1=\mathrm{C} 7-\mathrm{N} 2$ & $\mathrm{~N} 1=\mathrm{C} 7-\mathrm{C} 2$ & $\mathrm{C} 1=\mathrm{C} 2-\mathrm{C} 7$ & $\mathrm{C} 2=\mathrm{C} 1-\mathrm{N} 3$ & $C=C-R$ & C-R-C \\
\hline \multicolumn{9}{|l|}{$1 \mathrm{c}$} \\
\hline Exp. (X-ray) & 106.5935 & 116.1158 & 112.4017 & 124.8926 & 120.7913 & 120.3941 & $* * *$ & $* * *$ \\
\hline B3LYP/6-31G* & 106.1522 & 124.6597 & 111.3640 & 125.7553 & 121.7366 & 120.1569 & $* * *$ & $* * *$ \\
\hline $\mathrm{HF} / 3-21+\mathrm{G}^{* *}$ & 104.9584 & 121.4868 & 111.7900 & 126.0054 & 120.8060 & 120.2485 & $* * *$ & $* * *$ \\
\hline $\mathrm{HF} / 6-31+\mathrm{G}^{* *}$ & 107.0643 & 123.2378 & 112.5370 & 125.7849 & 122.1104 & 121.1487 & $* * *$ & $* * *$ \\
\hline \multicolumn{9}{|l|}{ PT-1c } \\
\hline B3LYP/6-31G* & 105.0817 & 126.1108 & 107.2887 & 124.7610 & 119.6377 & 117.1229 & $* * *$ & $* * *$ \\
\hline $\mathrm{HF} / 3-21+\mathrm{G}^{* *}$ & 105.5527 & 128.6621 & 107.5041 & 125.3549 & 119.5923 & 117.5128 & $* * *$ & $* * *$ \\
\hline \multicolumn{9}{|l|}{ 2c } \\
\hline B3LYP/6-31G* & 106.7180 & 122.4888 & 111.5543 & 125.7409 & 121.4766 & 121.2745 & 124.8825 & 118.4073 \\
\hline $\begin{array}{l}\mathrm{HF} / 3-21+\mathrm{G}^{* *} \\
\text { PT-2c }\end{array}$ & 105.2682 & 121.5254 & 111.8965 & 126.0302 & 120.9046 & 120.7542 & 123.8623 & 121.7831 \\
\hline B3LYP/6-31G* & 104.7454 & 125.5165 & 107.1581 & 124.8423 & 119.3946 & 117.7673 & 125.8462 & 117.8497 \\
\hline $\mathrm{HF} / 3-21+\mathrm{G}^{* *}$ & 105.6144 & 128.4032 & 107.4508 & 125.3034 & 119.4982 & 118.1297 & 125.3752 & 121.0656 \\
\hline $\begin{array}{l}\mathrm{HF}-\mathrm{CIS} / 3-21+\mathrm{G}^{* *} \\
\text { 3c }\end{array}$ & 103.4158 & 129.3885 & 105.4056 & 128.9085 & 121.2042 & 114.3756 & 122.9447 & 123.1390 \\
\hline B3LYP/6-31G* & 106.2127 & 126.5788 & 111.2331 & 125.6875 & 122.0009 & 119.4066 & 124.5404 & 118.6034 \\
\hline $\mathrm{HF} / 3-21+\mathrm{G}^{* *}$ & 104.7907 & 121.8068 & 111.7372 & 126.0612 & 120.9876 & 119.8715 & 123.9671 & 121.8551 \\
\hline \multicolumn{9}{|l|}{ PT-3c } \\
\hline B3LYP/6-31G* & 104.9478 & 125.1491 & 107.1631 & 125.1870 & 120.2843 & 116.7350 & 123.0438 & 118.9816 \\
\hline $\mathrm{HF} / 3-21+\mathrm{G}^{* *}$ & 105.4491 & 128.2868 & 107.4384 & 125.3575 & 119.9632 & 116.9455 & 122.4126 & 122.5365 \\
\hline HF-CIS/3-21+G** & 103.1226 & 129.3344 & 105.2612 & 129.6449 & 121.3734 & 113.8468 & 123.8522 & 121.6336 \\
\hline
\end{tabular}


Table S21: Selected dihedral angles (deg) for geometry-optimized structures.

\begin{tabular}{lccccc}
\hline & C1=C2-C7=N1 & C1-N3-S1-C14 & C2=C1-N3-H1 & C2=C1-N3-S1 & C-C-R-C \\
\hline 1c & & & & & \\
Exp. (TPBI X-ray) & -28.6369 & 58.3200 & 16.7570 & -99.3287 & $* * *$ \\
B3LYP/6-31G* & -14.3049 & 61.8973 & 5.5371 & -135.1551 & $* * *$ \\
HF/3-21+G** & -34.5849 & 63.3314 & 43.3780 & -104.0157 & $* * *$ \\
HF/6-31+G** & -29.5788 & 62.4861 & 20.8482 & -119.4779 & $* * *$ \\
PT-1c & & & & & \\
B3LYP/6-31G* & 0.9484 & 78.9755 & -0.0820 & 177.6598 & $* * *$ \\
HF/3-21+G** & 0.4304 & 68.9480 & -0.5478 & 179.1041 & $* * *$ \\
HF-CIS/3-21+G** & -1.0350 & 75.0849 & 0.6165 & -174.3325 & $* * *$ \\
2c & & & & & \\
B3LYP/6-31G* & -18.6950 & 62.2590 & 11.1941 & -124.4791 & 0.9602 \\
HF/3-21+G** & -36.2217 & 63.0857 & 46.1640 & -102.8964 & -2.9879 \\
PT-2c & & & & & \\
B3LYP/6-31G* & 0.9541 & 76.1671 & 0.3251 & -179.5330 & 1.7040 \\
HF/3-21+G** & 0.4246 & 69.0548 & -0.5403 & 178.7270 & -0.6879 \\
HF-CIS/3-21+G** & -1.3360 & 74.9860 & 0.8136 & -174.6125 & -0.6172 \\
3c & & & & & \\
B3LYP/6-31G* & -10.8979 & 64.7720 & 3.2607 & -143.6046 & 0.6611 \\
HF/3-21+G** & -34.4178 & 63.3595 & 42.5621 & -104.4083 & -0.6280 \\
PT-3c & & & & & \\
B3LYP/6-31G* & 0.7977 & 79.8969 & -0.1733 & 178.2029 & -1.5617 \\
HF/3-21+G** & 0.6630 & 66.2540 & -0.5209 & 179.6290 & -2.4740 \\
HF-CIS/3-21+G** & -0.6787 & 73.8307 & 0.3454 & -174.9677 & -4.0890 \\
\hline & & & & & \\
\hline
\end{tabular}


Summary of Computed Photophysical Properties (Tables S22-S30)

All transition energies are reported in electron-volts $(\mathrm{eV})$ and wavenumbers $\left(\mathrm{cm}^{-1}\right)$ for easy comparison with experimental data. The corresponding oscillator strengths ( $f$ ), static $(\mu)$ and transition $\left(\mathrm{M}_{\mathrm{ge}}\right)$ dipole moments, as well as the change in state dipole moments $\left(\Delta \mu_{\mathrm{ge}}\right)$ are reported for $\mathbf{1} \mathbf{c}-\mathbf{3} \mathbf{c}$ at the indicated levels of theory. The dominant determinants which contribute to the CI description are also presented. Vertical transition energies (excitation and emission) computed using the INDO-SCI implemented in either ZINDO(Z) or Argus Lab (AL) are noted where necessary. Results from calculations invoking the SCRF solvent model considering methanol (MeOH) and water $\left(\mathrm{H}_{2} \mathrm{O}\right)$ implemented in Argus Lab are noted. " $H$ " corresponds to a HOMO level and " $L$ " corresponds to a LUMO level.

Table S22: Computed vertical excitation energies (eV) and photophysical properties for 1c-3c (INDO-SCI(Z)//DFT-RB3LYP/6-31G(d)).

\begin{tabular}{lccc}
\hline Parameter & 1c & 2c & 3c \\
\hline $\mathrm{S}_{0}-\mathrm{S}_{1}(\mathrm{eV})$ & 3.86 & 3.84 & 3.80 \\
$\mathrm{~S}_{0}-\mathrm{S}_{1}\left(\mathrm{~cm}^{-1}\right)$ & 31120 & 30999 & 30637 \\
$f$ & 0.545 & 0.508 & 0.642 \\
C.I. Composition & $\mathrm{H}->\mathrm{L}$ & $\mathrm{H}->\mathrm{L}$ & $\mathrm{H}->\mathrm{L}$ \\
$\mu_{\mathrm{g}}(\mathrm{D})$ & $\mathrm{H}->\mathrm{L}+5$ & & \\
$\mu_{\mathrm{e}}(\mathrm{D})$ & 11.00 & 11.95 & 12.37 \\
$\Delta \mu_{\mathrm{ge}}(\mathrm{D})$ & 11.83 & 12.46 & 12.75 \\
$\mathrm{M}_{\mathrm{ge}}(\mathrm{D})$ & 0.83 & 0.51 & 0.38 \\
\hline
\end{tabular}

Table S23: Computed vertical excitation energies (eV) and photophysical properties for 1c-3c (INDO-SCI(Z)//RHF/3-21+G(d,p)).

\begin{tabular}{lccc}
\hline Parameter & 1c & 2c & 3c \\
\hline $\mathrm{S}_{0}-\mathrm{S}_{1}(\mathrm{eV})$ & 4.12 & 4.11 & 4.09 \\
$\mathrm{~S}_{0}-\mathrm{S}_{1}\left(\mathrm{~cm}^{-1}\right)$ & 33264 & 33185 & 32988 \\
$f$ & 0.319 & 0.331 & 0.438 \\
& $\mathrm{H}->\mathrm{L}$ & $\mathrm{H}->\mathrm{L}$ & $\mathrm{H}->\mathrm{L}$ \\
C.I. Composition & $\mathrm{H}->\mathrm{L}+4$ & $\mathrm{H}->\mathrm{L}+4$ & $\mathrm{H}->\mathrm{L}+4$ \\
& $\mathrm{H}-1->\mathrm{L}+6$ & $\mathrm{H}-1->\mathrm{L}+6$ & $\mathrm{H}-1->\mathrm{L}+6$ \\
$\mu_{\mathrm{g}}(\mathrm{D})$ & $\mathrm{H}-1->\mathrm{L}$ & $\mathrm{H}-1->\mathrm{L}$ & $\mathrm{H}-1->\mathrm{L}$ \\
$\mu_{\mathrm{e}}(\mathrm{D})$ & 10.28 & 9.38 & 8.62 \\
$\Delta \mu_{\mathrm{ge}}(\mathrm{D})$ & 11.33 & 9.68 & 9.57 \\
$\mathrm{M}_{\mathrm{ge}}(\mathrm{D})$ & 1.05 & 0.30 & 0.95 \\
& 4.51 & 4.60 & 5.31 \\
\hline
\end{tabular}


Table S24: Computed vertical excitation energies $(\mathrm{eV})$ and photophysical properties for 1c-3c (INDO-SCI(AL)//RHF/3-21+G(d,p)).

\begin{tabular}{lccc}
\hline Parameter & 1c & 2c & 3c \\
\hline $\mathrm{S}_{0}-\mathrm{S}_{1}(\mathrm{eV})$ & 4.12 & 4.10 & 4.07 \\
$\mathrm{~S}_{0}-\mathrm{S}_{1}\left(\mathrm{~cm}^{-1}\right)$ & 33216 & 33095 & 32834 \\
$f$ & 0.355 & 0.360 & 0.492 \\
& $\mathrm{H}->\mathrm{L}$ & $\mathrm{H}->\mathrm{L}$ & $\mathrm{H}->\mathrm{L}$ \\
& $\mathrm{H}->\mathrm{L}+5$ & $\mathrm{H}->\mathrm{L}+5$ & $\mathrm{H}->\mathrm{L}+5$ \\
C.I. Composition & $\mathrm{H}-1->\mathrm{L}+6$ & $\mathrm{H}-1->\mathrm{L}+6$ & $\mathrm{H}-1->\mathrm{L}+6$ \\
& $\mathrm{H}-1->\mathrm{L}$ & $\mathrm{H}-1->\mathrm{L}$ & $\mathrm{H}-1->\mathrm{L}$ \\
& & $\mathrm{H}-2->\mathrm{L}$ & \\
$\mu_{\mathrm{g}}(\mathrm{D})$ & 12.70 & 11.60 & 11.03 \\
$\mu_{\mathrm{e}}(\mathrm{D})$ & 13.95 & 11.97 & 12.06 \\
$\Delta \mu_{\mathrm{ge}}(\mathrm{D})$ & 1.25 & 0.37 & 1.03 \\
$\mathrm{M}_{\mathrm{ge}}(\mathrm{D})$ & 4.77 & 4.81 & 5.65 \\
\hline
\end{tabular}

Table S25: Computed vertical excitation energies (eV) and photophysical properties for 1c-3c (INDO-SCI(AL) SCRF $\mathrm{H}_{2} \mathrm{O} / / \mathrm{RHF} / 3-21+\mathrm{G}(\mathrm{d}, \mathrm{p})$ ).

\begin{tabular}{lccc}
\hline Parameter & 1c & 2c & 3c \\
\hline $\mathrm{S}_{0}-\mathrm{S}_{1}(\mathrm{eV})$ & 4.07 & 4.10 & 4.04 \\
$\mathrm{~S}_{0}-\mathrm{S}_{1}\left(\mathrm{~cm}^{-1}\right)$ & 32823 & 33051 & 32567 \\
$f$ & 0.373 & 0.365 & 0.494 \\
& $\mathrm{H}->\mathrm{L}$ & $\mathrm{H}->\mathrm{L}$ & $\mathrm{H}->\mathrm{L}$ \\
C.I. Composition & $\mathrm{H}->\mathrm{L}+5$ & $\mathrm{H}->\mathrm{L}+5$ & $\mathrm{H}->\mathrm{L}+5$ \\
& $\mathrm{H}-1->\mathrm{L}+6$ & $\mathrm{H}-1->\mathrm{L}+6$ & $\mathrm{H}-1->\mathrm{L}+6$ \\
$\mu_{\mathrm{g}}(\mathrm{D})$ & $\mathrm{H}-1->\mathrm{L}$ & $\mathrm{H}-1->\mathrm{L}$ & $\mathrm{H}-1->\mathrm{L}$ \\
$\mu_{\mathrm{e}}(\mathrm{D})$ & 14.82 & 13.49 & 12.77 \\
$\Delta \mu_{\mathrm{ge}}(\mathrm{D})$ & 16.44 & 13.99 & 14.15 \\
$\mathrm{M}_{\mathrm{ge}}(\mathrm{D})$ & 1.62 & 0.50 & 1.38 \\
\hline
\end{tabular}


Table S26: Computed vertical excitation energies $(\mathrm{eV})$ and photophysical properties for 1c-3c (INDO-SCI(AL) SCRF MeOH//RHF/3-21+G(d,p)).

\begin{tabular}{lccc}
\hline Parameter & 1c & 2c & 3c \\
\hline $\mathrm{S}_{0}-\mathrm{S}_{1}(\mathrm{eV})$ & 4.07 & 4.10 & 4.04 \\
$\mathrm{~S}_{0}-\mathrm{S}_{1}\left(\mathrm{~cm}^{-1}\right)$ & 32834 & 33051 & 32567 \\
$f$ & 0.373 & 0.365 & 0.494 \\
& $\mathrm{H}->\mathrm{L}$ & $\mathrm{H}->\mathrm{L}$ & $\mathrm{H}->\mathrm{L}$ \\
C.I. Composition & $\mathrm{H}->\mathrm{L}+5$ & $\mathrm{H}->\mathrm{L}+5$ & $\mathrm{H}->\mathrm{L}+5$ \\
& $\mathrm{H}->1->\mathrm{L}+6$ & $\mathrm{H}-1->\mathrm{L}+6$ & $\mathrm{H}-1->\mathrm{L}+6$ \\
$\mu_{\mathrm{g}}(\mathrm{D})$ & $\mathrm{H}-1->\mathrm{L}$ & $\mathrm{H}-1->\mathrm{L}$ & $\mathrm{H}-1->\mathrm{L}$ \\
$\mu_{\mathrm{e}}(\mathrm{D})$ & 14.75 & 13.43 & 12.72 \\
$\Delta \mu_{\mathrm{ge}}(\mathrm{D})$ & 16.36 & 13.92 & 14.09 \\
$\mathrm{M}_{\mathrm{ge}}(\mathrm{D})$ & 1.61 & 0.49 & 1.37 \\
\hline
\end{tabular}

Table S27: Computed vertical emission energies (eV) and photophysical properties for 1c-3c (INDO-SCI(Z)//RHF-CIS/3-21+G(d,p)).

\begin{tabular}{lccc}
\hline Parameter & 1c & 2c & 3c \\
\hline $\mathrm{S}_{1}-\mathrm{S}_{0}(\mathrm{eV})$ & 2.61 & 2.53 & 2.66 \\
$\mathrm{~S}_{1}-\mathrm{S}_{0}\left(\mathrm{~cm}^{-1}\right)$ & 21067 & 20366 & 21414 \\
$f$ & 0.452 & 0.464 & 0.447 \\
C.I. Composition & $\mathrm{H}->\mathrm{L}$ & $\mathrm{H}->\mathrm{L}$ & $\mathrm{H}->\mathrm{L}$ \\
$\mu_{\mathrm{g}}(\mathrm{D})$ & 13.16 & 14.45 & 14.90 \\
$\mu_{\mathrm{e}}(\mathrm{D})$ & 9.69 & 11.48 & 11.44 \\
$\Delta \mu_{\mathrm{ge}}(\mathrm{D})$ & -3.47 & -2.97 & -3.46 \\
$\mathrm{M}_{\mathrm{ge}}(\mathrm{D})$ & 6.75 & 6.96 & 6.66 \\
\hline
\end{tabular}

Table S28: Computed vertical emission energies (eV) and photophysical properties for 1c-3c (INDO-SCI(AL)//RHF-CIS/3-21+G(d,p)).

\begin{tabular}{lccc}
\hline Parameter & 1c & 2c & 3c \\
\hline $\mathrm{S}_{1}-\mathrm{S}_{0}(\mathrm{eV})$ & 2.63 & 2.54 & 2.67 \\
$\mathrm{~S}_{1}-\mathrm{S}_{0}\left(\mathrm{~cm}^{-1}\right)$ & 21221 & 20499 & 21476 \\
$f$ & 0.447 & 0.462 & 0.429 \\
C.I. Composition & $\mathrm{H}->\mathrm{L}$ & $\mathrm{H}->\mathrm{L}$ & $\mathrm{H}->\mathrm{L}$ \\
$\mu_{\mathrm{g}}(\mathrm{D})$ & 13.36 & 14.37 & 15.17 \\
$\mu_{\mathrm{e}}(\mathrm{D})$ & 11.14 & 12.79 & 12.72 \\
$\Delta \mu_{\mathrm{ge}}(\mathrm{D})$ & -2.22 & -1.58 & -2.45 \\
$\mathrm{M}_{\mathrm{ge}}(\mathrm{D})$ & 6.69 & 6.92 & 6.52 \\
\hline
\end{tabular}


Table S29: Computed vertical emission energies (eV) and photophysical properties for 1c-3c (INDO-SCI(AL) SCRF $\mathrm{H}_{2} \mathrm{O} / / \mathrm{RHF}-\mathrm{CIS} / 3-21+\mathrm{G}(\mathrm{d}, \mathrm{p})$ ).

\begin{tabular}{lccc}
\hline Parameter & 1c & 2c & 3c \\
\hline $\mathrm{S}_{1}-\mathrm{S}_{0}(\mathrm{eV})$ & 2.68 & 2.57 & 2.72 \\
$\mathrm{~S}_{1}-\mathrm{S}_{0}\left(\mathrm{~cm}^{-1}\right)$ & 21638 & 20711 & 21971 \\
$f$ & 0.447 & 0.453 & 0.444 \\
C.I. Composition & $\mathrm{H}->\mathrm{L}$ & $\mathrm{H}->\mathrm{L}$ & $\mathrm{H}->\mathrm{L}$ \\
$\mu_{\mathrm{g}}(\mathrm{D})$ & 15.72 & 16.99 & 17.52 \\
$\mu_{\mathrm{e}}(\mathrm{D})$ & 13.32 & 15.06 & 15.16 \\
$\Delta \mu_{\mathrm{ge}}(\mathrm{D})$ & -2.40 & -1.93 & -2.36 \\
$\mathrm{M}_{\mathrm{ge}}(\mathrm{D})$ & 6.65 & 6.84 & 6.58 \\
\hline
\end{tabular}

Table S30: Computed vertical emission energies (eV) and photophysical properties for 1c-3c (INDO-SCI(AL) SCRF MeOH//RHF-CIS/3-21+G(d,p)).

\begin{tabular}{lccc}
\hline Parameter & 1c & 2c & 3c \\
\hline $\mathrm{S}_{1}-\mathrm{S}_{0}(\mathrm{eV})$ & 2.68 & 2.57 & 2.72 \\
$\mathrm{~S}_{1}-\mathrm{S}_{0}\left(\mathrm{~cm}^{-1}\right)$ & 21620 & 20702 & 21957 \\
$f$ & 0.446 & 0.453 & 0.444 \\
C.I. Composition & $\mathrm{H}->\mathrm{L}$ & $\mathrm{H}->\mathrm{L}$ & $\mathrm{H}->\mathrm{L}$ \\
$\mu_{\mathrm{g}}(\mathrm{D})$ & 15.64 & 16.91 & 17.45 \\
$\mu_{\mathrm{e}}(\mathrm{D})$ & 13.25 & 14.99 & 15.08 \\
$\Delta \mu_{\mathrm{ge}}(\mathrm{D})$ & -2.39 & -1.92 & -2.37 \\
$\mathrm{M}_{\mathrm{ge}}(\mathrm{D})$ & 6.65 & 6.84 & 6.58 \\
\hline
\end{tabular}




\section{Summary of Plotted Densities and Isosurfaces}

All figures were generated using data from Q-Chem plot outputs. Electron attachment corresponds to the LUMO while detachment corresponds to the HOMO.

Table S31: Selected molecular orbital isosurfaces and electron attachment/detachment densities for 1c-3c (HF-CIS/3-21+G(d,p) optimized geometries).

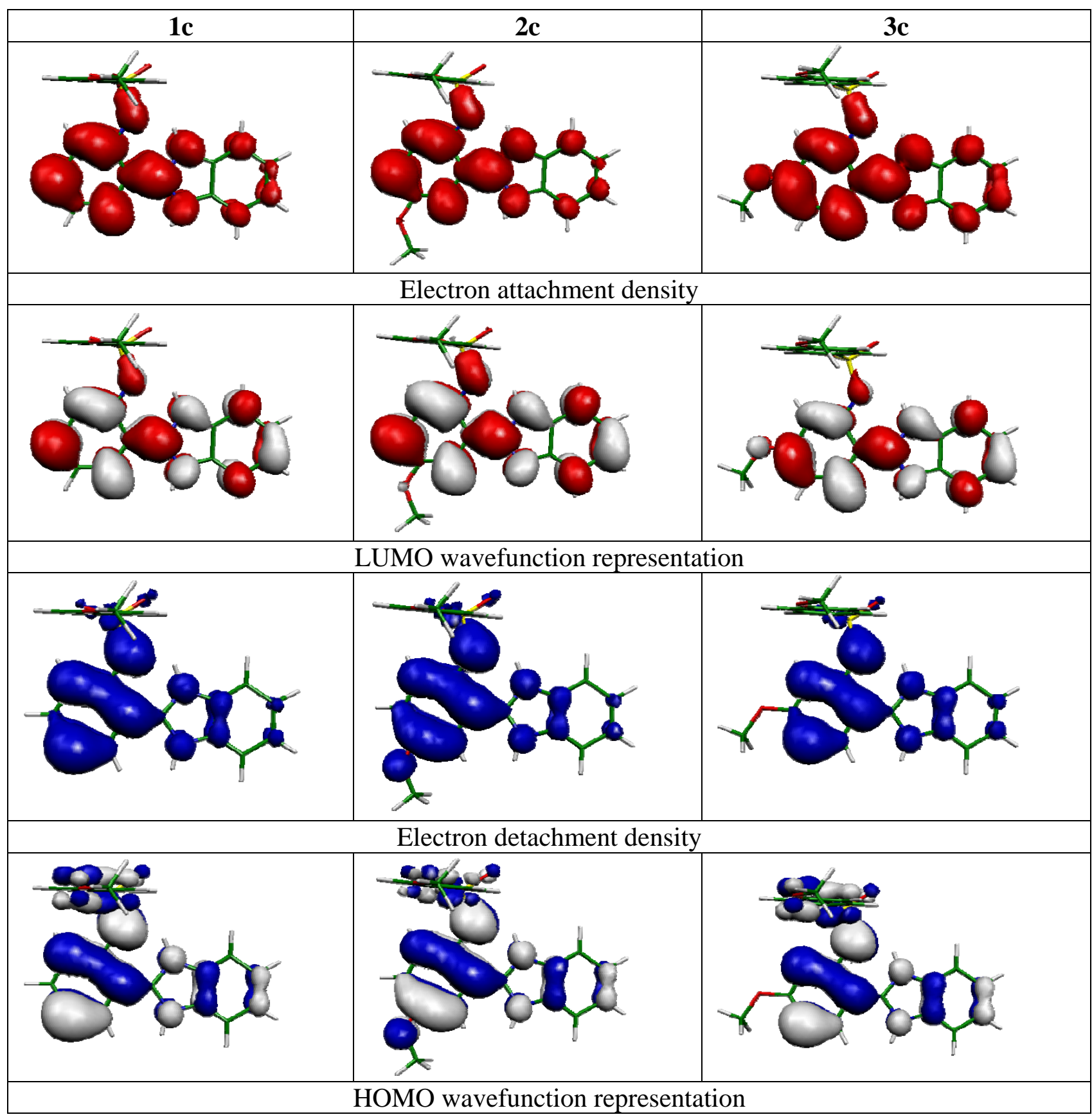

